\title{
Interference effect of body shadow in action control
}

\author{
Arnaud Badets ${ }^{1 *}$, Lucette Toussaint ${ }^{1,2}$, \\ Yannick Blandin ${ }^{1,2}$ and Christel Bidet-Ildei ${ }^{1,2}$
}

${ }^{1}$ Centre de Recherches sur la Cognition et l'Apprentissage, UMR-7295

Centre National de la Recherche Scientifique (CNRS), France

${ }^{2}$ University of Poitiers, France

*Corresponding author:

Arnaud Badets

Centre de Recherches sur la Cognition et l'Apprentissage

CNRS, UMR-7295

Maison des Sciences de l'Homme et de la Société

Bât A5; 5, rue Théodore Lefebvre

86000 Poitiers-FRANCE

Tel.: +330632833870

Fax: +330549454616

E-mail: arnaud.badets@univ-poitiers.fr

Short Title: Shadow in action

Keywords: Cast shadow, automatic imitation, hand action 


\begin{abstract}
Observing actions performed by other persons can subsequently influence our own motor behaviors. However, it is unknown whether the shadows cast by such actions can also have an impact on the observers' action. Here we show that the mere observation of a cast shadow can influence imitative behaviors. Specifically, participants were shown a hand picture and its associated cast shadow in a neutral position. In a "compatible trial", the hand and shadow that followed were turned in the same open or closed direction, while in an "incompatible trial" the hand and shadow were turned in different directions. We contrasted two experimental conditions: 1) "Hand-Shadow" in which participants observed a hand and its cast shadow (the hand covered the shadow), 2) "Hand-Hand" in which participants observed a hand and another black hand (the shadow covered the hand). The participants' task was to imitate (i.e., by closing or opening their own hands) the hand or the cast shadow action. For both conditions, results revealed interference (i.e., longer response latencies) for incompatible trials. This suggests for the first time that the mere observation of a cast shadow of a hand can influence imitative behaviors. However, time courses of the response latencies revealed that imitative effect in the Hand-Shadow condition was different than the imitative effect in the Hand-Hand condition. Therefore, we suggest considering the cast shadow of an action as an important feature during motor control for humans.
\end{abstract}


Observing another person performing actions can influence our subsequent motor behaviors (Bandura, 1986; Mattar \& Gribble, 2005; Heyes, 2011). For example, Press and colleagues (2005) asked participants to open or close their right hands as soon as they perceived a closing or opening movement of a human or a robotic hand. The results revealed a stronger interference when participants observed a human hand. Specifically, the response latencies increased drastically when participants were required to produce an incongruent movement (e.g., closing hand movement while observing an opening action) rather than a congruent one. This finding reveals a tendency to automatically imitate the actions of others (Badets \& Pesenti, 2010; Stürmer, Aschersleben \& Prinz, 2000; Heyes, 2011, for a review). Interestingly, scholars have discovered that, at a neurophysiological level, such imitative behaviors are governed by an "action observation network" (AON) that includes the premotor cortex, the primary motor cortex, and the inferior parietal lobule (see Press, 2011 for a review of the AON). This AON is active when people observe and then imitate the actions of others, and this activation is specific to human actions (Press et al. 2005; Press, Gillmeister \& Heyes, 2007; Press, 2011). However, during the observation of real life actions, the cast shadows of these actions are sometimes present in the visual scenes. For instance, during a soccer game with a shiny sun, the different body shadows projected on the ground could be processed as important features to take into account for different strategic behaviors like the precise positions of players between them. Yet, it is unknown whether such information can indeed influence motor performance of observers. The general aim of the present study was to test whether the cast shadow of an action can interfere with the mechanism of automatic imitation.

Cast shadows of objects are projected on a remote surface and provide important information (e.g., dispositions of objects in space) for visual processing (see Mamassian, Kill \& Kersten, 1998 for this definition). From this perspective, cast shadows of objects represent fundamental cues for interaction with the environment (Galfano \& Pavani, 2005). For instance, Bonfiglioli, Pavani and 
Castiello (2004, Experiment 2) have found that if a cast shadow is dissimilar from the natural cast shadow of an object, the movement trajectory for a reach-to-grasp action toward this object is affected. To the authors, such findings suggested that the cast shadow of an object is processed during the control of an action. Interestingly, it has recently been suggested that a cast shadow of a body could provide a crucial tool for recognizing a person. For instance, the shadow of a person perfectly depicts the shape of the body, and it has been shown that individual identification is improved when judgments are based on the shadow cast on the ground in daylight in comparison to the conventional method in which identification is based on the actual body area (Iwashita, Stoica \& Kurazume, 2010). Pavani and Castiello (2004) have also suggested that such a cast shadow of the body could be an additional cue for sensorimotor representation. Specifically, they suggested that body shadows are involved in the elaboration of the "body schema" (see also Galfano \& Pavani, 2005), which is a core representation of the positions of the parts of the body in space (Whiteley, Spence \& Haggard, 2008). Note that the body schema is an important system for movement commands that includes, at a neuronal level, the parietal and the premotor cortex (Giummarra, Gibson, Georgiou-Karistianis \& Bradshaw, 2007), which are both areas of the AON (Press, 2011).

The AON also includes the primary motor cortex, which has been found to be a key locus in the observation of cast shadows of movements (Alaerts, Aggelpoel, Swinnen \& Wenderoth, 2009). In this study, Alaerts and colleagues asked participants to observe a hand or the shadow of this hand depicting a rhythmically adducted-abducted movement of the index finger. During this movement observation, transcranial magnetic stimulation was applied over the primary motor cortex of the participants while motor-evoked potentials (MEPs) were recorded from their index finger muscle. The results revealed that the MEP amplitude increased for the observation of the shadow or the real finger movements. The authors suggested that the observer's motor system (i.e., the AON; Press, 2011) is active during the observation of a shadow depicting a movement or 
the observation of the real action. Therefore, the mere observation of a shadow can have a strong influence on the motor system of observers (see also Sartori \& Castiello, 2013 for similar interpretation).

However, based on these studies of shadow movement, it is unknown whether the mere observation of a cast shadow of another person can also have an influence on subsequent movements of observers. To the best of our knowledge, the single piece of evidence for this assumption comes from an animal study. Indeed, animals such as crayfish can use the movement of a shadow projected on the ground to execute anti-predator behaviors (Liden \& Herberholz, 2008). For instance, if a shadow moves towards the animal at high velocity, the crayfish will stop its movement, likely in an attempt to reduce its visibility. This finding suggests that observing a shadow linked to an action of another can have a strong influence on subsequent motor behaviors. Consequently, based on the AON for humans, it could be hypothesized that if a hand movement and the shadow of this hand can influence the motor system (Alaerts et al. 2009), we should find an automatic imitation effect from the hand, and also the cast shadow movement.

In the present study, participants were asked to observe two stimuli and to open or close their right hand in response to a change in only one stimulus. The stimuli were a hand and its cast shadow in a neutral position and the hand and its cast shadow turned into an opening or closing position (Figure 1, panel A). For the Hand-Shadow condition (HS), the cast shadow was projected on a remote surface and did not cover the hand (Mamassian et al. 1998). With this stimulus configuration, we expected to provide participants with an adequate correspondence between the hand and its cast shadow. Mamassian (2004) suggested the following for the cast shadow information: "in order to use that information, our visual system has first to segment regions in the image, decide that these regions are potential shadows rather than, say, ink blots, and then match these shadow candidates with objects in the scene. We call this last processing stage the "shadow correspondence problem"." (p.1279). For the Hand-Hand condition (HH), the shadow 
covered the hand and, consequently, could not be interpreted as a cast shadow but, more likely, as another hand. Indeed, we reasoned that if the shadow was presented as covering the hand, the shadow correspondence would be impossible for the participants (Casati, 2012; Mamassian, 2004). Consequently, the participants should interpret this stimulus as a second hand because, despite its black color, a cast shadow cannot cover a hand under natural conditions. To ensure that our assumptions were correct, a post-experimental interview about these stimuli was recorded by the experimenter at the end of the study.

The participants were instructed to follow the action of one of two stimulus positions. For instance, in the first block of the HS condition, the participant was asked to follow the firstposition stimulus. Consequently, this participant's task was to open his/her hand when the hand stimulus (i.e., the first-position stimulus) turned in the open direction. Note that participants were instructed not to pay attention to the second-position stimulus (i.e., the cast shadow). However, this second-position stimulus could turn into a compatible or incompatible direction (opening or closing, respectively). For a second block of trials, the participants were instructed to follow the second-position stimulus (i.e., the cast shadow), and, consequently, the compatible and incompatible directions came from the hand action.

From the theoretical background developed in the present paper and our experimental paradigm, we expected to confirm four main hypotheses. First, we expected that participants would interpret (assessed through the post-experimental interview) the two stimuli differently, that is, a hand and its cast shadow in the HS condition and two hands in the HH condition. Indeed, as emphasized by the definition of a cast shadow (Casati, 2012; Mamassian et al. 1998), it is highly improbable that participants recognize as a shadow a stimulus that covers the hand. Second, for the $\mathrm{HH}$ condition we predicted a classical interference effect. Indeed, participants should interpret both stimuli as two hands, and as suggested by Press and colleagues (2005; see also Stürmer, et al. 2000; Heyes, 2001) the mere observation of a hand mimicking an action can induce a strong 
automatic imitation even when the second stimulus is task-irrelevant (see also Brass, Bekkering \& Prinz, 2001 for finger movements). Third, and most importantly, in the HS condition, we predicted an interference effect from the cast shadow action as hypothesized by Alearts et al. (2009). Indeed, based on the different findings regarding the cast shadow of the body (Galfano \& Pavani, 2005; Iwashita et al. 2010; Pavani \& Castiello, 2004; Pavani \& Galfano, 2007), we expected that the mere observation of a cast shadow of a hand action could have a strong influence on the motor system of the observers. Specifically, our data should reveal an interference effect in the imitative behavior in the condition in which the participants followed the hand regardless of whether they were asked to ignore the cast shadow. Finally, if the participants interpreted a hand with its cast shadow for the HS condition, the two stimuli could represent a single action from a single hand (a correct shadow correspondence). Conversely, if the participants interpreted two hands from the $\mathrm{HH}$ condition, then the two stimuli could represent two different actions. Consequently, based on the biological specificity of the AON, we predicted that the two biological hands of the HH condition could interfere more strongly than a single hand with its cast shadow could. To test this last hypothesis, we have used an analysis on the distribution from the fastest to the slowest responses times (see Catmur \& Heyes, 2011; Brass et al. 2001 Experiment 2; Press et al. 2005 for similar analysis in imitation paradigms). In this perspective, Brass and colleagues found that the compatibility effect during an imitation task of a biological stimulus was larger for slower responses (see also Press et al. 2005). This finding is interpreted as the imitation processes underlying compatibility effect becomes more implicated over time. Consequently, we could expect the same effect when the biological specificity of the AON is fully engaged, that is, in the $\mathrm{HH}$ condition.

\section{Method}

Participants. Thirty-six (34 right-handed and 2 left-handed) French-speaking students from the University of Poitiers (mean age: $19.8 \pm 1$ year; 25 males) participated in this study for course 
credit and were distributed equally in each group through hand-laterality. All participants had normal or corrected-to-normal vision, were unaware of the experimental goals, and provided informed and signed consent. The experiment was non-invasive and was performed in accordance with the ethical standards established by the Declaration of Helsinki.

Stimuli, apparatus and task. The stimuli were right male hands presented in their natural colors. The neutral and opening position involved a distance of 3.5 and $9.5 \mathrm{~cm}$, respectively, between the index finger and thumb. In the closing position, the thumb was shown joined with all other fingers. Length (L) and Height $(\mathrm{H})$ of the neutral, opening and closing positions occupied a window of $5 \mathrm{~cm} \times 10 \mathrm{~cm}, 10.5 \mathrm{~cm} \times 8.5 \mathrm{~cm}$ and $5 \mathrm{~cm} \times 9 \mathrm{~cm}$ respectively which corresponded to the actual visual angles $4.8^{\circ}(\mathrm{L}) \times 9.7^{\circ}(\mathrm{H}), 10.02^{\circ}(\mathrm{H}) \times 8.1^{\circ}(\mathrm{L})$, and $4.8^{\circ}(\mathrm{H}) \times 8.6^{\circ}(\mathrm{L})$ at a viewing distance of $60 \mathrm{~cm}$. The cast shadow was the same hand figure presented in grey color mimicking a real shadow (Figure 1, panel A). The apparatus consisted of a PC computer equipped with a $43.17 \mathrm{~cm}$ (diagonal) screen and a manual-response device composed of two microswitches (see Figure 1, panel B). The microswitches were connected to the PC computer, and a customized E-Prime ${ }^{\circledR}$ program controlled the experimental procedure and stored the raw data for offline analysis. Using the manual-response device, the participant's task was to open or close his or her right hand when an opening or closing hand or cast shadow appeared on the screen. Figure 1, panel B, shows the participant's task. For the neutral position (middle picture), microswitches A (bottom small strip) and B (the top small strip) are in the "on" and "off" positions, respectively. For the opening position (top picture), microswitch A is released and turns into the "off" position. For the closing position (bottom picture), microswitch B is squeezed and turns into the "on" position. Note that from the neutral position, the PC computer stored a valid manual response with its respective response latency from the "off" and "on" position of the microswitches for the opening and closing movements of the participant, respectively. 


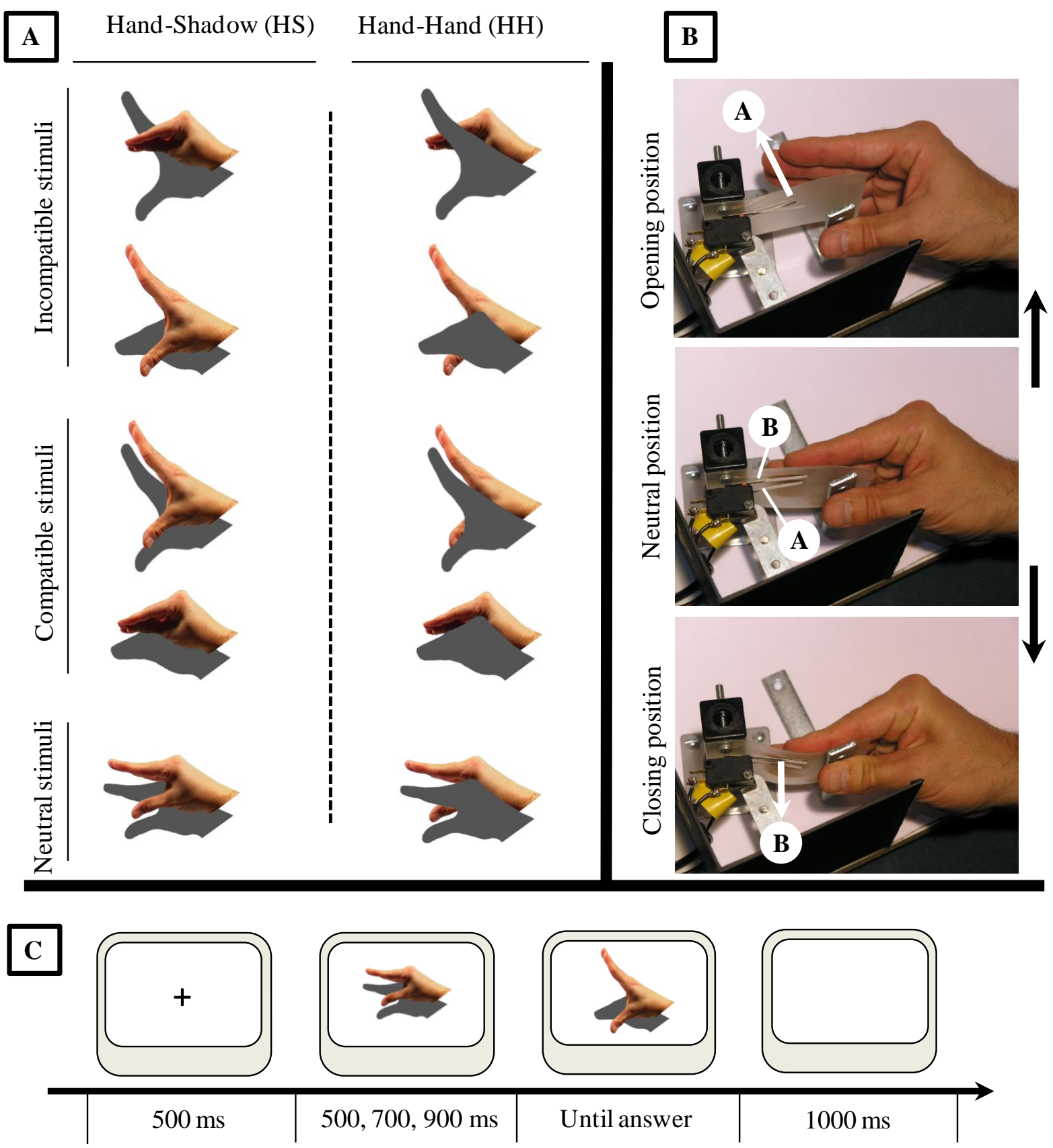

Figure 1. (A) Neutral, compatible and incompatible stimuli representing the hand and its cast shadow for the HS and HH conditions. (B) Manual-response device in the opening, neutral and closing position; A and B represent the microswitches, the white arrows represent the directions of the opening and closing action from the neutral position, and the black arrows represent these actions from the neutral to the opening and closing positions. (C) Temporal sequence of events for a trial in the HS condition. For each trial, neutral stimuli were displayed and changed into compatible or incompatible stimuli. The same sequence was used for the $\mathrm{HH}$ condition. 
Procedure. The participants sat in front of the computer screen, and the manual-response device. After participants were given the instructions and some handles for familiarization with the manual-response device, the experiment began. Participants were randomly assigned to one of the experimental conditions (HH or HS). In each experimental condition, each participant attended two sessions in which his/her task was to follow the first-position or the second-position stimulus. Each trial began with the presentation of a fixation cross for $500 \mathrm{~ms}$. Next, an HS or $\mathrm{HH}$ condition in a neutral position (Figure 1, panel A) was presented for random intervals of 500, 700, or $900 \mathrm{~ms}$ to prevent anticipation. Then, the neutral picture changed into an opening or closing picture. The beginning of the change was the imperative stimulus to which the participants responded; the final state of the change was displayed until the response was detected (Figure 1, panel C). The next trial began $1000 \mathrm{~ms}$ after the participant's response. The second stimulus was either compatible or incompatible with the first. For instance, when participants were required to follow the first-position stimulus in the HS condition, the compatibility came from the shadow, that is, the second-position stimulus (see Figure 1, Panel A and Panel C for an example of an incompatible trial). To avoid attentional anticipations of the position of the shadow or the hand, half of the shadow stimuli were presented slightly under the hand stimuli, whereas the other half the shadow stimuli were presented slightly over the hand stimuli (see Figure 1A). Both the stimulus presentations and the compatible and incompatible trials were presented randomly during the experiment.

In total, 2 blocks of 120 trials were presented to the participants. For the first and second blocks, the participants were required to follow the first-position and the second-position stimulus, respectively. This rule was counterbalanced across participants. For each block, 60 compatible trials and 60 incompatible trials were presented. At the end of the experiment, participants answered the following question: "what did the stimuli on the screen represent?" This question was given in French (i.e., “que représentaient les stimuli à l'écran de l'ordinateur?”). 
Participants were instructed to respond as simply as possible, and the experimenter noted these responses on a sheet of paper.

Design. The independent variables included CONDITIONS (Hand-Hand vs. Hand-Shadow) as a between-groups factor, and RULES (first-position vs. second-position) and COMPATIBILITY (compatible vs. incompatible) as within-group factors.

Dependent variables. We used the mean response latency (in ms) as dependent variables (Press et al. 2005, 2007). The response latency was calculated from the presentation on the screen of the apparent closing or opening action of the hand or the shadow to the opening or closing movement of the participant. In our analyses, we excluded trials with errors related to the COMPATIBILITY (averaging 2.3\%). Response latencies were then submitted to an analysis of variance (ANOVA), and post-hoc comparisons were computed using Duncan's technique. All significant effects were reported at $p<0.05$ (unless otherwise noted).

For the question asked at the end of the experiment, a chi-square test was conducted to assess whether different types of responses were chosen in the two different conditions (the HS and the HH condition). Specifically, we attributed a numeric value of " 1 " for each response that included one of the following statements: "I have seen two hands" or "I have seen a hand and its shadow" or "I have seen a hand and an object". Note that the first and second answers correspond to the $\mathrm{HH}$ and HS condition, respectively. It is worth noting that the last response was never given by participants.

\section{Results}

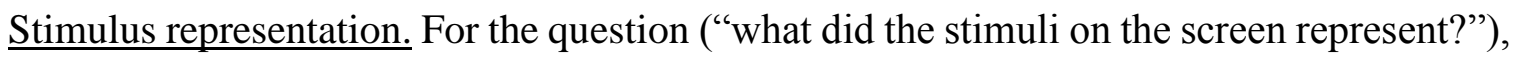
sixteen participants in the $\mathrm{HH}$ condition reported that the stimuli presented on the screen were two hands, that is, one in a black color and another in a real color. Two participants reported that the stimuli were a hand and a shadow because a shadow is generally black in color. For the HS 
condition, fifteen participants reported that the stimuli were a real hand with its cast shadow, but three participants reported two different hands because the two stimuli did not move in the same direction. A chi-square test indicated a significant difference in responses between the $\mathrm{HH}$ and the HS conditions $\left(\chi^{2}[1]=18.8 ; p<.0001\right)$. This analysis revealed that participants more frequently interpreted the $\mathrm{HH}$ condition as two hands (88.9\%) and the $\mathrm{HS}$ condition as a hand and its cast shadow $(83.3 \%)$.

Response latency. The dependent variable was analyzed in a 2 CONDITIONS (Hand-Hand $v s$. Hand-Shadow) X 2 RULES (first-position vs. second-position) X 2 COMPATIBILITY (compatible vs. incompatible) ANOVA with the last two factors defined as within-group independent variables. The analysis revealed a main effect only for COMPATIBILITY $(\mathrm{F}[1,34]=106.2, p<$ $\left..00001, \eta_{\mathrm{p}}^{2}=.75\right)$. This effect indicated that for both conditions, the participants responded faster for compatible than for incompatible trials (see Figure 2). No main effects from CONDITIONS $(\mathrm{F}[1,34]<1)$ or RULES $(\mathrm{F}[1,34]=1.8, p=.18)$ and no interaction between CONDITIONS and COMPATIBILITY $(\mathrm{F}[1,34]=1.2, p=.27)$, or other factors $(\mathrm{Fs}[1,22]<1)$ were observed.

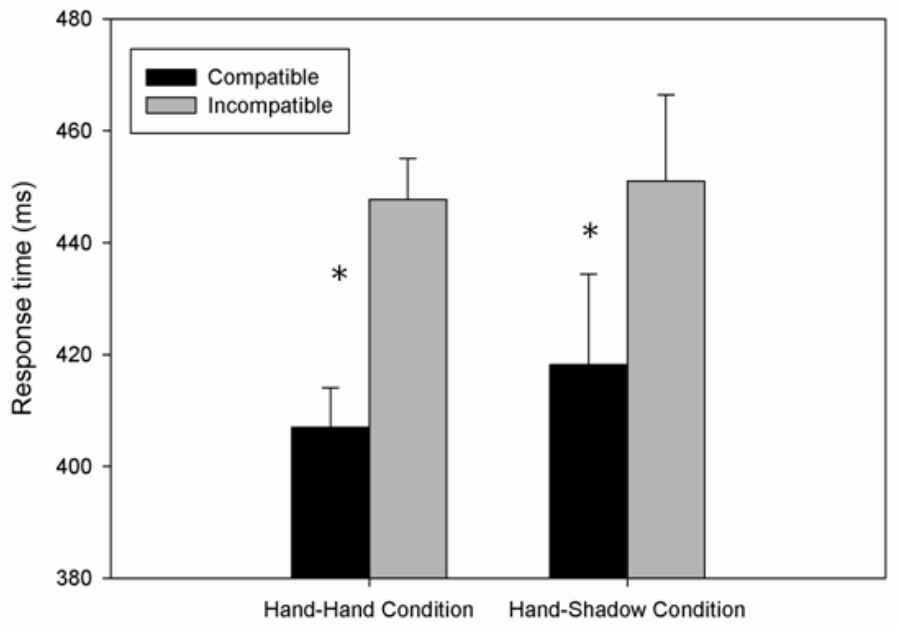

Figure 2. The mean response latencies for the Hand-Hand condition and the Hand-Shadow condition as a function of compatibility. * indicates significant differences and error bars represent 1 Standard error. 
To explore whether the compatibility effect had different temporal dynamics for the $\mathrm{HH}$ and HS condition, a distribution analysis was performed on response latencies (Ratcliff, 1979). For this purpose, we divided the response distribution of each participant into ten "bins" ranging from the fastest to the slowest response times. The dependent variable was then analyzed in a 2 CONDITIONS (Hand-Hand vs. Hand-Shadow) X 2 COMPATIBILITY (compatible vs. incompatible) X 10 BINS ANOVA with the last two factors defined as within-group independent variables. The analysis revealed a main effect for COMPATIBILITY $\left(\mathrm{F}[1,34]=95.4, p<.00001, \eta_{\mathrm{p}}^{2}=.73\right)$, BINS $\left(\mathrm{F}[9,306]=409.3, p<.00001, \eta_{\mathrm{p}}^{2}=.92\right)$, a significant CONDITIONS X BINS interaction $(\mathrm{F}[9,306]$ $\left.=1.9, p<.04, \eta_{\mathrm{p}}^{2}=.05\right)$, and a significant COMPATIBILITY X BINS interaction $(\mathrm{F}[9,306]=2.6, p$ $\left.<.005, \eta_{\mathrm{p}}^{2}=.07\right)$. Most importantly, the analysis revealed a significant CONDITIONS $\mathrm{X}$ COMPATIBILITY X BINS interaction $\left(\mathrm{F}[9,306]=3.02, p<.001, \eta_{\mathrm{p}}^{2}=.08\right)$. For this last interaction (see Figure 3), post-hoc analysis revealed that for the bins 1, 2, and 3, there is no compatibility effect for both conditions. The compatibility effect appeared for both conditions and grew up from bins 4 to 9 . However, for the last bin, there is a significant compatibility effect only for the $\mathrm{HH}$ condition. In other words, for both conditions there is an identical compatibility effect for bins 4-9, but this effect vanished to the slowest response time for the HS condition. 

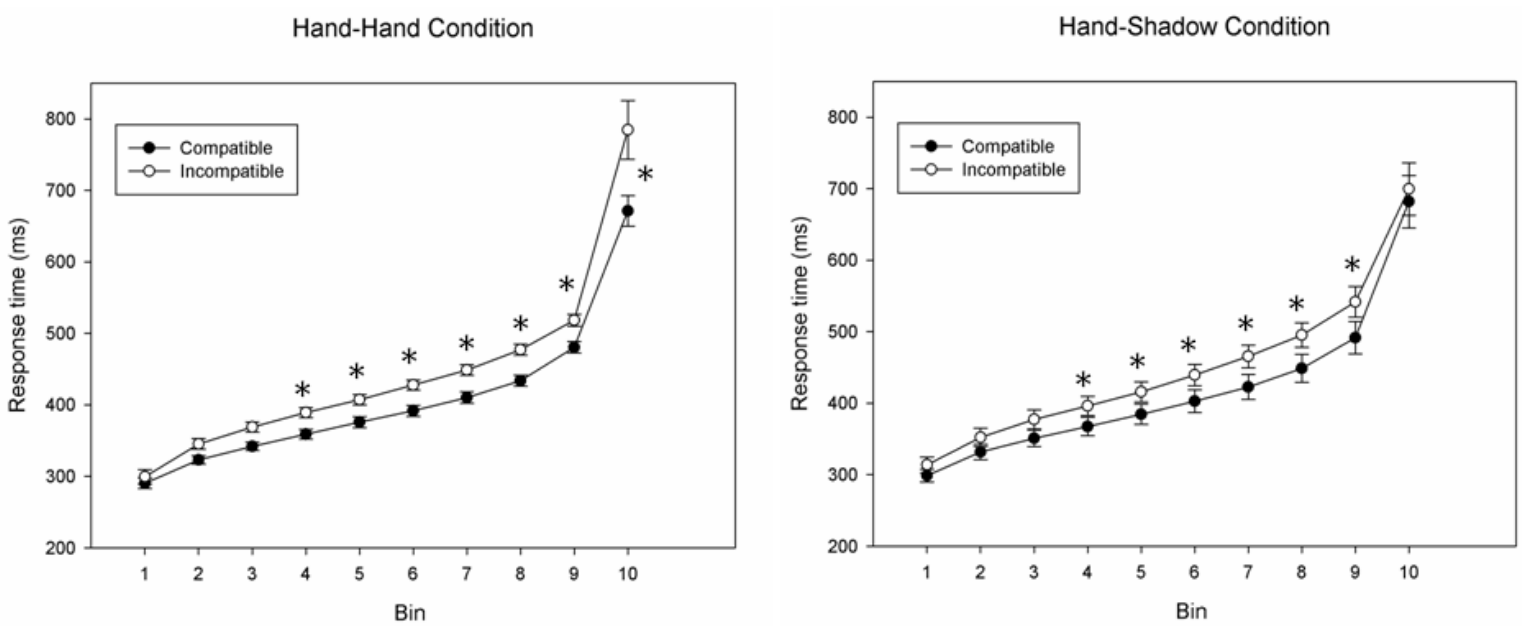

Figure 3. Mean response latencies in each bin as a function of compatibility for the Hand-Hand and Hand-Shadow condition. * indicates significant differences, and error bars represent 1 standard error.

\section{Discussion}

The general aim of this study was to assess whether the mere observation of a cast shadow of a hand action could influence the observers' own hand movement. In the HS condition, participants were required to observe a hand that performed a movement of opening or closing its fingers. In some trials, the cast shadow of this hand followed the hand (compatible trials), and in other trials, the hand and cast shadow moved in the opposite way (incompatible trials). The response times clearly indicate that the mere presentation of a cast shadow can influence the observers' capacity to follow the moving hand. Specifically, response times were longer for incompatible than for compatible trials. The distribution analysis revealed that this effect was present for the HS condition only for the bins 4-9. For the HH condition, the effect was also present for the slowest responses (i.e., the last bin). Altogether, these findings confirmed the strong effect of biological stimuli during an imitation task (Brass et al. 2001; Press et al. 2005), but more importantly, that a body shadow associated with its action can be interpreted as an important feature for the action control of observers. 
The present study confirmed that for the HH condition and for the HS condition, the participant perceived two hands or a hand and its cast shadow, respectively (see the stimulus representation section). This result suggests that when a hand covered the shadow that is, a shadow projected on a remote surface, the participants interpreted this stimulus as a cast shadow (Mamassian et al. 1998, see Casati, 2012; Mamassian, 2004 for this shadow correspondence effect). In contrast, when the shadow covered the hand, this stimulus was interpreted as another hand. Moreover, the factor RULES (first-position $v s$. second-position) did not interact with other factors $(F s<1)$. This result indicates that in the HS condition, the shadow influenced the observer's action to the same extent than the hand stimulus, at least for the bins 4-9. In other words, there was no more interference from the shadow or the hand stimulus. Consequently, we assume that for the HS condition, participants perceived a single hand. Our interpretation is that, if this single hand displayed incongruent information (from the hand itself or its cast shadow) depending of the action to-be-performed, an inference occurred in the $\mathrm{AON}$ of the observers.

To summarize the present findings, we confirmed the main hypotheses presented in the introduction section. First, through the configuration of the stimuli (Casati, 2012; Mamassian, 2004), the participants interpreted the HH and HS conditions as two hands or as a hand and its cast shadow, respectively. Second, the configuration of two hands (i.e., HH), or a hand with its cast shadow afforded interference as suggested by the AON (Alaerts et al. 2009; Press, 2011). Thus, the observation of cast shadow of an action can influence the motor control of observers. However, the distribution analysis of response times revealed that the interference was always present for the slowest response latencies only for the $\mathrm{HH}$ condition. Consequently, the hand and its cast shadow seem weaker to create the same level of interference in the motor system of the observers than the HH condition. Based on the biological specificity of the AON (Press, 2011), this last finding accord with the assumption that the interference should be stronger from two hands than a single one. 
It is worth noting that our results are in accordance with the finding that in an automatic imitation paradigm, incompatible trials induce stronger interference than compatible trials do (Heyes, 2011; Press et al. 2005, 2007; Stürmer et al. 2000). We found this interference effect in the HH and the HS condition for the bins 4-9 which represented a speed distribution for response times from 350 to $500 \mathrm{~ms}$. For comparable speeds of response times, other studies have found the same interference effect for different imitation paradigms (Brass et al. 2001, Experiment 2; Catmur \& Heyes, 2001; Press et al. 2005). Our present design is also comparable to the study by Stürmer et al. (2000) in which participants had to grasp or to spread their hand apart after the perception of a color change in the model's hand. In this study, the color change is the imperative stimulus, and the model's hand can be congruent (compatible trials) or incongruent (incompatible trials) depending on the action to be performed. Though our study utilized a different stimulus (a hand position), we also found longer response latencies in incompatible trials than in compatible trials. This finding for the $\mathrm{HH}$ condition suggests that we have perfectly replicated the classical effect of the automatic imitation domain, that is, the human tendency to copy the actions of others (Heyes, 2011; Press, 2011).

However, using the same design for the HS condition, the interference effect (reflected by the distribution analysis) was less because the slowest response times (the last bin) were the same for both compatible and incompatible trials. To interpret this, we suggested above that when participants perceive a cast shadow with a single hand, the $\mathrm{AON}$ is less active than the perception of two hands (i.e., the $\mathrm{HH}$ condition). Another interpretation could be that perceiving a hand and its cast shadow as we presented them in the incompatible trials (e.g., the hand closing and the shadow opening) can be highly irrelevant for the observers because this situation is impossible in a real life situation. For participants in the HS condition, this improbable situation may have afforded no significant interference, which, in turn, did not influence to the capacity to copy the model's action, especially during longer response times (around $700 \mathrm{~ms}$ ). In other words, 
participants took advantage of their longer response times in order to reject or to inhibit such improbable information from the AON. In agreement with this assumption of different levels of interference, Costantini and colleagues (2005) have found specific activation in sensorimotor parietal areas during the observation of biomechanically impossible movements of fingers.

However, our current findings should be interpreted with caution and require further empirical evidences to fully confirm our assumptions about the role of a body shadow in motor control. Indeed, as suggested by an anonymous reviewer, our general interpretation about the distinction between the $\mathrm{HH}$ and the HS condition is only based on the last bin of the distribution analysis and a post-experimental interview about the stimuli. Specifically, it is difficult to rule out entirely the possibility that during the experimental task, the participants did not process areal hand for the black stimulus in the HS condition, especially for bins 1-9 which showed exactly the same pattern of results for both HH and HS conditions. Accordingly, the present study gives a weak but preliminary support for the general claim about the specific role of cast shadows in the imitative behaviour of observer. So far, more studies are needed in order to fully confirm whether the AON is less active because participants process one single hand with its associated shadow for the HS condition in comparison to two hands for the $\mathrm{HH}$ condition.

Finally, as suggested by Pavani and Castiello (2004; see also Galfano \& Pavani, 2005), the main role of a body shadow is most likely to afford additional information for the sensorimotor representation and, specifically, the body schema. For these authors, the cast shadow of a body can be crucial in isolating the self from the environment and, especially, in constructing the gap between personal and extrapersonal space (Pavani \& Castiello, 2004). This suggestion is in accordance with the view that the AON is also involved in distinguishing the self from the other and, more precisely, in understanding the actions of others (Neal \& Kilner, 2010; Rizzolatti \& Sinigaglia, 2010, for a review). From an evolutionary perspective, Pavani and Galfano (2007) suggested the following regarding body shadows: “They have presumably accompanied humans 
throughout evolution providing important cues for self-recognition, as well as recognition of bodies of other approaching living creatures.” (p. 86). Similarly, Rizzolatti and Sinigaglia (2010) suggested of the AON that "Such motor-based understanding seems to be a primary way in which individuals relate to one another, as shown by its presence not only in humans and monkeys, but also in evolutionarily distant species, such as swamp sparrows and zebra finches." (p. 273). Consequently, based on the present automatic imitation paradigm, which has been used to test the AON (Heyes, 2011; Press, 2011), we suggest that the interference effect found for the HS condition is the first behavioral evidence of this cast shadow account system for action control in human observers (see Liden \& Herberholz, 2008, for a behavioral interpretation in crayfish). In line with this evolutionary perspective, it has recently been suggested that parts of the AON could be involved in understanding the actions of others to develop defensive behaviors for observers of different species (Blanchard, Griebel, Pobbe, \& Blanchard, 2011). Defensive behaviors could also develop in humans through cast shadow processing inside the AON.

\section{Conclusion}

Body shadow can have a specific influence on action control. The observation of such a shadow can involve the primary motor cortex of observers (Alaerts et al. 2009) and, at a behavioral level, can present important information for the formation of sensorimotor representation (Pavani \& Castiello, 2004). The present study follows this line of research and demonstrates for the first time that shadow is also functionally implicated in the motor system of observers during imitation. Theoretically, this implication suggests that the body shadow is likely an important feature for the AON system. However, additional empirical studies at a behavioral and neurophysiological level are needed to fully encompass our preliminary findings about the precise role of shadow in actions. 


\section{Acknowledgments}

We are grateful to Cédric Bouquet and Yves Almecija (CNRS-UMR-7295) for their valuable comments regarding the editing of the pictures and the device used in the present study. 


\section{References}

Alaerts, K., Van Aggelpoel, T., Swinnen, S. P., \& Wenderoth, N. (2009). Observing shadow motions: resonant activity within the observer's motor system? Neuroscience Letters, 461, 240-244. doi: 10.1016/j.neulet.2009.06.055.

Badets, A., \& Pesenti, M. (2010). Creating number semantics through finger movement perception. Cognition, 115, 46-53. doi: 10.1016/j.cognition.

Bandura, A. (1986). Social foundations of thought and action: A social cognitive theory. Englewood Cliffs, NJ: Prentice-Hall.

Blanchard, D. C., Griebel, G., Pobbe, R., \& Blanchard, R. J. (2011). Risk assessment as an evolved threat detection and analysis process. Neuroscience and Biobehavioral Reviews, 35, 991-8. doi: 10.1016/j.neubiorev.2010.

Bonfiglioli, C., Pavani, F., \& Castiello, U. (2004). Differential effects of cast shadows on perception and action. Perception, 33, 1291-1304. doi: 10.1068/p5325.

Brass, M., Bekkering, H., \& Prinz, W. (2001). Movement observation affects movement execution in a simple response task. Acta Psychologica, 106, 3-22. doi: http://dx.doi.org/10.1016/S0001-6918(00)00024-X.

Casati, R. (2012). Some varieties of shadow illusions: Split shadows, occluded shadows, stolen shadows, and shadows of shadows. Perception, 41, 357-360. doi:10.1068/p7156web.

Catmur, C. \& Heyes, C. (2011). Time course analyses confirm independence of imitative and spatial compatibility. Journal of Experimental Psychology: Human Perception and Performance, 37, 409-421. doi: 10.1037/a0019325.

Costantini, M., Galati, G., Ferretti, A., Caulo, M., Tartaro, A., Romani, G. L., \& Aglioti, S. M. (2005). Neural systems underlying observation of humanly impossible movements: an fmri study. Cerebral Cortex, 15, 1761-1767. doi: 10.1093/cercor/bhi053. 
Galfano, G., \& Pavani, F. (2005). Long-lasting capture of tactile attention by body shadows. Experimental Brain Research, 166, 518-527.doi:10.1007/s00221-005-2392-9.

Giummarra, M. J., Gibson, S. J., Georgiou-Karistianis, N., \& Bradshaw, J. L. (2007). Central mechanisms in phantom limb perception: the past, present and future. Brain Research Reviews, 54, 219-232. doi:10.1016/j.brainresrev.2007.01.009.

Heyes, C. M. (2011). Automatic imitation. Psychological Bulletin, 137, 463-483. doi: $10.1037 / \mathrm{a} 0022288$.

Iwashita, Y., Stoica, A., \& Kurazume, R. (2010). Person Identification using Shadow Analysis. In Labrosse, F., Zwiggelaar, R., Liu, Y., \& Tiddeman, B. editors, Proceedings of the British Machine Vision Conference, 35, 1-10. BMVA Press. doi:10.5244/C.24.35.

Liden, W. H., \& Herberholz, J. (2008). Behavioral and neural responses of juvenile crayfish to moving shadows. Journal of Experimental Biology, 211, 1355-1361. doi: 10.1242/jeb.010165.

Mamassian, P., Knill, D. C., \& Kersten, D. (1998). The perception of cast shadows. Trends in Cognitive Sciences, 2, 288-295. doi:10.1016/S1364-6613(98)01204-2.

Mamassian, P. (2004). Impossible shadows and the shadow correspondence problem. Perception, 33, 1279-1290. doi: 10.1068/p5280.

Mattar, A. A. G., \& Gribble, P. L. (2005). Motor learning by observing. Neuron, 46, 153-160. doi: 10.1016/j.neuron.2005.02.009.

Neal, A., \& Kilner, J. M. (2010). What is simulated in the action observation network when we observe actions? European Journal of Neuroscience, 32, 1765-1770. doi: 10.1111/j.14609568.2010.07435.x. 
Pavani, F., \& Castiello, U. (2004). Binding personal and extrapersonal space through body shadows. Nature Neuroscience, 7, 13-14. doi:10.1038/nn1167.

Pavani, F., Galfano, G. (2007). Self-attributed body-shadows modulate tactile attention. Cognition, 104, 73-88. doi: http://dx.doi.org/10.1016/j.cognition.2006.05.007.

Press, C. (2011). Action observation and robotic agents: learning and anthropomorphism. Neuroscience and Biobehavioral Reviews, 35, 1410-1418. doi: 10.1016/j.neubiorev.2011.03.004.

Press, C., Bird, G., Flach, R. \& Heyes, C. (2005). Robotic movement elicits automatic imitation. Cognitive Brain Research, 25, 632-640. doi: 10.1016/j.cogbrainres.2005.08.020.

Press, C., Gillmeister, H., \& Heyes, C. (2007). Sensorimotor experience enhances automatic imitation of robotic action. Proceedings of the Royal Society B: Biological Sciences, 274, 2639-2644. doi: 10.1098/rspb.2007.0774.

Ratcliff, R. (1979). Group reaction time distributions and an analysis of distribution statistics. Psychological Bulletin, 86, 446-461.

Rizzolatti, G., \& Sinigaglia, C. (2010). The functional role of the parieto-frontal mirror circuit: interpretations and misinterpretations. Nature Reviews Neuroscience, 11, 264-74. doi: $10.1038 / \mathrm{nrn} 2805$.

Stürmer, B., Aschersleben, G., \& Prinz, W. (2000). Correspondence effects with manual gestures and postures: a study of imitation. Journal of Experimental Psychology: Human Perception \& Performance, 26, 1746-1759. doi: 10.1037//0096-1523.26.6.1746.

Sartori, L., \& Castiello, U. (2013). Shadows in the mirror. Neuroreport, 24, 63-67. doi: 10.1097/WNR.0b013e32835c6e6a. 
Shadow in action 23

Whiteley, L., Spence, C., \& Haggard, P. (2008). Visual processing and the bodily self. Acta Psychologica, 127, 129-136. doi: 10.1016/j.actpsy.2007.03.005. 